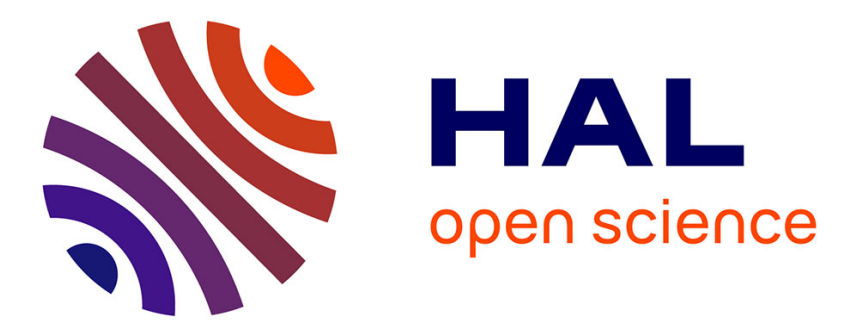

\title{
Combined fitting of alternative and direct susceptibility curves of assembled nanostructures
}

\author{
A Hillion, Matthias Pauly, A Tamion, F Tournus, Matthias Hillenkamp, \\ Benoit Pichon, Sylvie Begin-Colin, Véronique Dupuis
}

\section{- To cite this version:}

A Hillion, Matthias Pauly, A Tamion, F Tournus, Matthias Hillenkamp, et al.. Combined fitting of alternative and direct susceptibility curves of assembled nanostructures. Journal of Applied Physics, 2012, 112 (12) (Article number : 123902), 10.1063/1.4768837 . hal-02530426

\section{HAL Id: hal-02530426 \\ https://hal.science/hal-02530426}

Submitted on 2 Apr 2020

HAL is a multi-disciplinary open access archive for the deposit and dissemination of scientific research documents, whether they are published or not. The documents may come from teaching and research institutions in France or abroad, or from public or private research centers.
L'archive ouverte pluridisciplinaire HAL, est destinée au dépôt et à la diffusion de documents scientifiques de niveau recherche, publiés ou non, émanant des établissements d'enseignement et de recherche français ou étrangers, des laboratoires publics ou privés. 


\section{Combined fitting of alternative and direct susceptibility curves of assembled nanostructures}

A. Hillion, M. Pauly, A. Tamion, F. Tournus, M. Hillenkamp, B. P. Pichon, S. Begin-Colin, and V. Dupuis

Citation: Journal of Applied Physics 112, 123902 (2012); doi: 10.1063/1.4768837

View online: https://doi.org/10.1063/1.4768837

View Table of Contents: http://aip.scitation.org/toc/jap/112/12

Published by the American Institute of Physics

\section{Articles you may be interested in}

Accurate determination of the magnetic anisotropy in cluster-assembled nanostructures

Applied Physics Letters 95, 062503 (2009); 10.1063/1.3200950

Demixing in cobalt clusters embedded in a carbon matrix evidenced by magnetic measurements Journal of Applied Physics 110, 063904 (2011); 10.1063/1.3638035

Static and dynamic magnetic properties of spherical magnetite nanoparticles Journal of Applied Physics 94, 3520 (2003); 10.1063/1.1599959

Superparamagnetism

Journal of Applied Physics 30, S120 (1959); 10.1063/1.2185850

Anisotropic ferromagnetic polymer: A first step for their implementation in microfluidic systems AIP Advances 6, 056604 (2016); 10.1063/1.4943927

Self consistent measurement and removal of the dipolar interaction field in magnetic particle assemblies and the determination of their intrinsic switching field distribution

Journal of Applied Physics 111, 083914 (2012); 10.1063/1.4704397

\section{PHYSICS TODAY}

MANAGER'S GUIDE WHITEPAPERS

\section{READ NOW}

PRESENTED BY

Accelerate R\&D with
Multiphysics Simulation 


\title{
Combined fitting of alternative and direct susceptibility curves of assembled nanostructures
}

\author{
A. Hillion, ${ }^{1}$ M. Pauly, ${ }^{2}$ A. Tamion, ${ }^{1}$ F. Tournus, ${ }^{1}$ M. Hillenkamp, ${ }^{3}$ B. P. Pichon, ${ }^{2}$ \\ S. Begin-Colin, ${ }^{2}$ and V. Dupuis ${ }^{1}$ \\ ${ }^{1}$ Laboratoire de Physique de la Matière Condensée et Nanostructures, UMR CNRS 5586, Université Lyon 1, \\ Bât. L. Brillouin, 6 rue Ada Byron, 69622 Villeurbanne cedex, France \\ ${ }^{2}$ Institut de Physique et de Chimie des Matériaux de Strasbourg (UMR CNRS/UdS/ECPM 7504), 23 rue du \\ Loess BP43, 67034 Strasbourg cedex 2, France \\ ${ }^{3}$ Laboratoire de Spectrométrie Ionique et Moléculaire, UMR CNRS 5579, Université Lyon 1, Bât. Kastler, \\ 6 rue Ada Byron, 69622 Villeurbanne cedex, France
}

(Received 20 September 2012; accepted 7 November 2012; published online 17 December 2012)

\begin{abstract}
Experimental ac-susceptibility curves at different frequencies $(0.1 \mathrm{~Hz} \leq f \leq 1 \mathrm{kHz})$ were performed on samples prepared by physical and chemical pathways. By combining the triple fit method and a careful analysis of ac-experimental curves, we demonstrate an unambiguous and consistent determination method of both the magnetic particle size distribution and anisotropy for diluted granular nanostructures of magnetic clusters. Specifically, we highlight the importance of the size distribution in the determination of the magnetic anisotropy constant as well as the low relevance of the deduced parameters by considering alternative measurements alone. (c) 2012 American Institute of Physics. [http://dx.doi.org/10.1063/1.4768837]
\end{abstract}

\section{INTRODUCTION}

Magnetic nanoparticles (NPs) are currently the subject of intense research because of their potential applications in future ultrahigh-density magnetic-storage media ${ }^{1}$ but also in biomedical applications. ${ }^{2,3}$ They are considered as the building blocks of the future nanotechnological devices through bottom-up approaches. The knowledge of the properties of NPs (particle size distribution and anisotropy) is of primary importance for the further development of all these applications. Combinations of several methods are often necessary to determine the specific NPs properties which may be affected by dipolar interactions. Indeed when the NPs size decreases, they become single domain, view as macrospin and their potential is limited by the trade-off between the blocking of the particles' magnetic moments and the thermal excitation, leads to the so-called superparamagnetic (SP) limit. ${ }^{4}$ The reversal of the magnetic moment of an isolated single domain particle with a volume $V$ and an effective uniaxial anisotropy constant $K_{\text {eff }}$ over an energy barrier $\Delta E$ is characterized by the Neel relaxation time, ${ }^{5}$

$$
\tau=\tau_{0} \exp \left(\frac{\Delta E}{k_{B} T}\right)
$$

where $\tau_{0}$ is a pre-exponential relaxation time factor in the range $10^{-12}-10^{-9} \mathrm{~s},{ }^{6} k_{B}$ is the Boltzmann constant, and $\Delta E=K_{\text {eff }} V$.

The anisotropy barrier $\Delta E$ is a determinative factor in the static and dynamic behavior of NPs. Therefore, the knowledge of $\Delta E$ is very important to predict and understand the magnetic properties of NPs. In a system formed of fine particles with a size distribution, one recurring problem is the unambiguous determination of the nanoparticle magnetic size distribution as well as of their anisotropy constant. ${ }^{7} \mathrm{~A}$ possible way is to measure the size distribution from microscopy observations. However, this method can sometimes be unreliable and the structural size obtained from microscopy may be different from the effective magnetic size. ${ }^{8}$

In this paper, we first review a method to obtain anisotropy barrier distributions from the temperature, time, and magnetic field dependent magnetization/susceptibility $(\chi)$ measurements. The fit procedure is based on the alternative susceptibility $\chi_{\mathrm{ac}}$ and includes size distributions effects. The most accurate $K_{\text {eff }}$ determination is achieved by also considering static susceptibility and high temperature $m(H)$ fits. ${ }^{9}$ In addition, the obtained values of $\tau_{0}$ are discussed. As an illustration of the high applicability of this careful analysis on magnetic nanoparticles, this model is applied to two different benchmark samples of well-separated cobalt nanoparticles (prepared by a physical way) and $\mathrm{Fe}_{3-\mathrm{x}} \mathrm{O}_{4}$ nanoparticles (synthesized by chemical way).

\section{THEORETICAL DESCRIPTION}

The two most common protocols used to detect the signature of the blocked to superparamagnetic regime crossover in NPs assemblies are the static (dc) susceptibility measurements versus temperature: measured after zero field cooling (ZFC) and field cooling (FC). Assuming a random orientation of the easy axes and that the applied magnetic field is small enough to be in the linear response regime, we can write the ZFC magnetic moment for the two extreme behaviors

$$
\begin{aligned}
& m_{b}=N_{t o t} \frac{\mu_{0} H \mu^{2}}{3 \Delta E} \text { in the blocked regime, } \\
& m_{0}=N_{t o t} \frac{\mu_{0} H \mu^{2}}{3 k_{B} T} \text { in the superparamagnetic regime, }
\end{aligned}
$$

where $\mu$ is the magnetic moment of one single NP and $N_{t o t}$ is the total number of particles.

In the "two state model," the total susceptibility is given by the sum of the contributions of the superparamagnetic 
and blocked particles weighted by the appropriate size distribution function. This model has several limitations as listed in Refs. 10 and 11. A more elaborate model, called "progressive crossover model" has been recently developed $^{10,11}$ and provides a continuous analytical formula which can be used to describe the ZFC curve of NPs assembly. For a single volume,

$$
m_{Z F C}=m_{b} e^{-\nu \delta t}+m_{0}\left(1-e^{-\nu \delta t}\right),
$$

where $\nu=1 / \tau$ is the macrospin relaxation frequency (Eq. (1)) and $\delta t$ is an effective waiting time which depends on several parameters. ${ }^{10} \mathrm{~A}$ similar expression can be used for the FC, which may be considered as a ZFC with a different starting point in the case of a size distribution.

The ZFC/FC curves and the superparamagnetic $m(H)$ can be fitted using semi-analytical formulas. Moreover, it is important to note that the curves share some common quantities, in particular the magnetic size distribution (diameter probability density function) $\operatorname{PDF}(D)$ and the total number of NPs $\left(N_{t o t}\right)$. A simultaneous fit of the three experimental curves, the so-called "triple fit" procedure, is then subject to stringent constraints so that any fortuitous agreement is very unlikely leading to an improved accuracy of the inferred results. The application of this model to characterize welldefined Co and CoPt NPs has proven the robustness of this technique. ${ }^{9,12-14}$ This procedure is a good way to test the usual underlying hypotheses and to put into evidence subtle effects such as an anisotropy constant dispersion in nanoalloys. $^{12}$

ZFC susceptibility curves can also be measured in the ac mode. In this case, after having cooled down the sample without any applied field, a small alternative magnetic field with a pulsation $\omega$ is applied. Then, the subsequent magnetic moment oscillating at the same $\omega$ pulsation is measured, as a function of temperature. The induced magnetic moment can be written as: $m=m^{\prime}-i m^{\prime \prime}$. A theoretical expression of the real and imaginary parts can be established ${ }^{15-18}$ and we have, for a single volume $V$

$$
\begin{gathered}
m^{\prime}=m_{b}+\frac{m_{0}-m_{b}}{1+\omega^{2} \tau^{2}}, \\
m^{\prime \prime}=\omega \tau \frac{m_{0}-m_{b}}{1+\omega^{2} \tau^{2}},
\end{gathered}
$$

where $m_{0}$ and $m_{b}$ corresponds, respectively, to the equilibrium (superparamagnetic) and blocked magnetic moments and $\tau$ is given by Eq. (1). Experimental curves can be fitted with the above theoretical expressions, by performing a numerical integration in order to take into account the particle size distribution. It is also possible, as for dc curves, to fit the real part by using a two state or "abrupt transition" model which assumes that a particle is either fully blocked or fully superparamagnetic. ${ }^{15}$ The peak temperature $T_{\max }$ should not be confused with the mean or median blocking temperature because it strongly depends on the shape of the size distribution.

Moreover, the imaginary part of the signal is insensitive to parasitic magnetic signals. And by varying the $\omega$ pulsa- tion, we have access to a quite wide range of timescales, which can be used to estimate the relaxation time. The finding of an unphysical value for $\tau_{0}$ enables to detect the presence of significant interactions in a NPs sample. ${ }^{6,19,20}$ Besides, it is also quite common to derive a single energy barrier value from the evolution of $T_{\max }$ with $\omega$, using an Arrhenius-type plot. We can write, for a single energy barrier (in this case $T_{\max } \sim T_{B}$ )

$$
\ln \frac{1}{\omega}=\ln \tau_{0}+\frac{K_{e f f} V}{k_{B} T_{\max }} .
$$

This means that a plot of $-\ln \omega$ as a function of $1 / T_{\max }$ should consist in a straight line where the slope is directly related to the magnetic anisotropy energy, while the crossing point with the $\mathrm{Y}$-axis corresponds to $\ln \tau_{0}$.

\section{EXPERIMENTAL ANALYSIS}

The first system under study is composed of cobalt nanoparticles synthesized by the low energy cluster beam deposition technique (LECBD) as detailed in Ref. 21. Cobalt nanoparticles around $3 \mathrm{~nm}$ in diameter are prepared in the gas phase and deposited fragmentation-free onto a Si substrate, together with a protective Au matrix. The independent choice of the cluster and matrix fluxes ratio allows tuning the particle concentration, necessary to avoid inter-particle interactions. The cluster diameter PDF before and after deposition closely follows a log-normal distribution

$$
P D F(D)=\frac{1}{\sigma \sqrt{2 \pi}} \frac{1}{D} \exp \left[-\frac{1}{2}\left(\frac{\ln \left(D / D_{m}\right)}{\sigma}\right)^{2}\right],
$$

where $D$ and $D_{m}$ are, respectively, the diameter and the median diameter of the NPs and $\sigma$ is the standard deviation. All the magnetic measurements have been performed using a superconducting interference device magnetometer (Quantum Design MPMS 5 XL).

We used the triple fit procedure ${ }^{9}$ in order to determine the effective cluster magnetic anisotropy $\left(K_{\text {eff }}\right)$ and the magnetic size distribution $\left(\operatorname{PDF}\left(D_{m a g}\right)\right)$. For the benchmark sample with Co nanoparticles embedded in gold matrix, we derive the following values from the triple fit (cf. Figure 1): a magnetic median diameter $D_{m, m a g}=3.4 \mathrm{~nm}$ with a standard deviation of $\sigma_{\text {mag }}=0.28$ and an effective anisotropy $K_{\text {eff }}=178 \mathrm{~kJ} \mathrm{~m}^{-3}$, with a relative error around $10 \%$. The size distribution derived from the triple fit is in complete agreement with TEM analysis. ${ }^{9}$

Typical thermal variations of the real part of the alternative susceptibility $\chi_{\mathrm{ac}}$ are shown in Fig. 2. As expected for a system of SP particles, a transition from the blocked to the $\mathrm{SP}$ regime is observed and the maximum temperature $\left(T_{\max }\right)$ is found to increase when increasing the applied field frequency. The $\chi_{\mathrm{ac}}$ curves have been fitted simultaneously at different frequencies using the log-normal size distribution deduced from TEM and triple fit. As can be seen in Fig. 1, experimental curves are well reproduced by fixing this value and we obtain a value of $K_{\text {eff }}=176 \pm 10 \mathrm{~kJ} \mathrm{~m}^{-3}$ which is in excellent agreement with the one deduced from triple fit. 


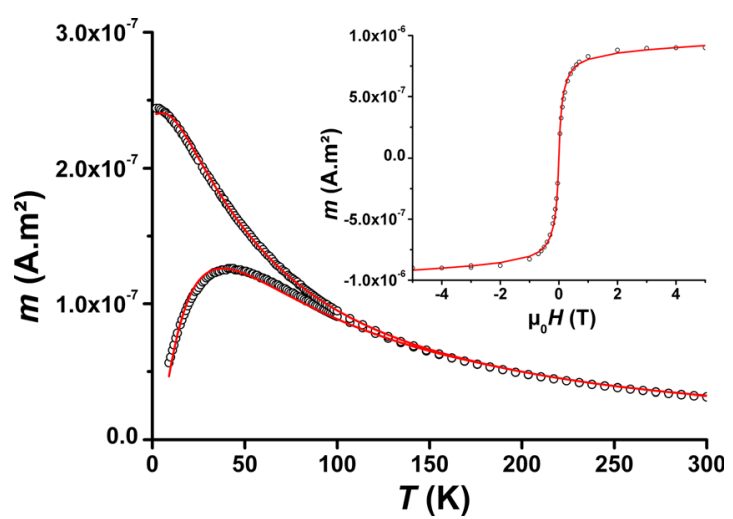

FIG. 1. ZFC/FC magnetization curves of the Co: Au sample (1\% vol.). The red lines correspond to the fit using the "triple fit." The $m(H)$ curve at $300 \mathrm{~K}$ is shown in inset.

Besides, from the fit of the entire curves, we can deduce $\tau_{0}=5 \times 10^{-11} \mathrm{~s}$ with a large uncertainty because the curves only weakly depend on $\tau_{0}$. Figure 2 (inset) presents the variation of $-\ln \omega$ versus $1 / T_{\max }$, which is commonly used to extract the magnetic anisotropy. ${ }^{22-27}$ Using this approach, the fit of this curve with a linear function allows determining the "average" energy barrier $\Delta E / k_{B}=1050 \mathrm{~K}$ and $\tau_{0}=2$ $\times 10^{-10} \mathrm{~s}$. The two methods give a very close $\tau_{0}$ in the range expected for a sample without interactions. We can also deduce $K_{\text {eff }}$ by using the relation $\Delta E=K_{\text {eff }} . V_{m}$, where $V_{m}$ is the mean volume of the particles. We obtain $K_{\text {eff }}$ $=590 \mathrm{~kJ} \mathrm{~m}^{-3}$, which is three times larger than the value deduced from dc and ac fits. In fact, this analysis does not take into account the size distribution and $T_{\max }$ does not reflect the blocking temperature of the mean size, which leads to an overestimated $K_{\text {eff }}$ value.

Interestingly, we have found that even for our benchmark system, the fit of the $\chi_{\mathrm{ac}}$ curve alone is not unambiguous to determine the particle size distributions. As an example, different log-normal PDFs with median diameters between 2.7 and $3.6 \mathrm{~nm}$ could equally reproduce the real part $m^{\prime}$ with different $K_{\text {eff }}$ (see Fig. 3); $\chi_{\text {ac }}$ curves seem to be not as sensitive to size distribution as the dc ZFC/FC curves. Notably, we underline that the merging of the ZFC and FC curves is the most sensitive regions to size dispersion.

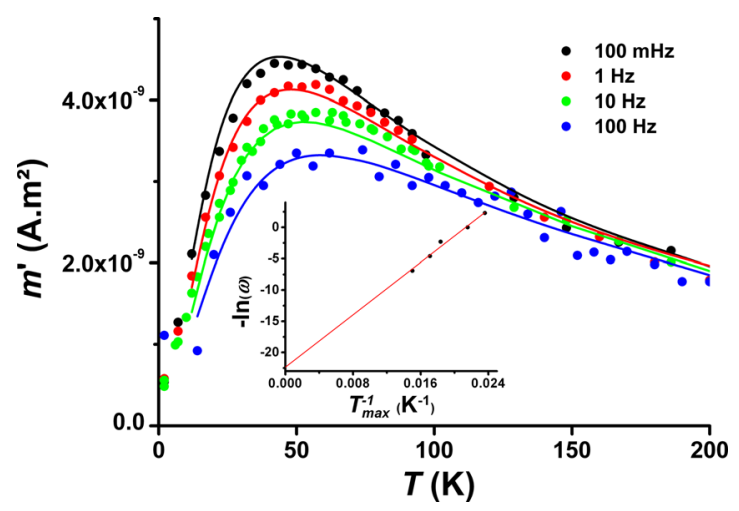

FIG. 2. Thermal variations of the real ac-magnetic moment $\left(\mathrm{m}^{\prime}\right)$ plotted for different frequencies for the Co: Au sample. The solid line corresponds to the fit described in the text, using PDF deduced from the "triple fit." Experimental variation of $-\ln \omega$ as function of $1 / \mathrm{T}_{\max }$ and fit using the Néel relaxation (Eq. (1)) with a constant $\tau_{0}$ (inset).

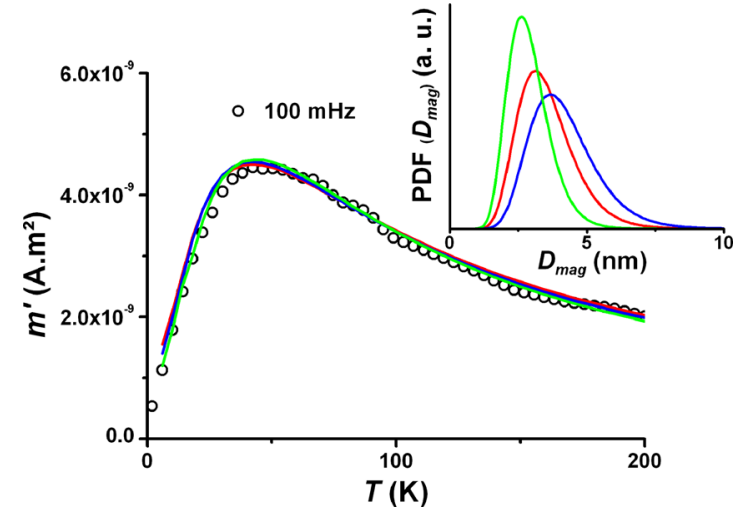

FIG. 3. Thermal variations of the real ac-magnetic moment $\left(m^{\prime}\right)$ at $100 \mathrm{mHz}$ of the Co: Au sample. The experimental curve can be nicely adjusted using Eqs. (3) and (6) with three different magnetic PDFs shown in the inset.

Now, we use our procedure for magnetic iron oxide nanoparticles synthesized by chemical way. The chemical method has the advantage to produce large quantities of NPs, thus ac-measurements are strongly facilitated. The imaginary part, hidden in the noise in the previous sample, is in this case exploitable. Spherical iron oxide nanoparticles of $\sim 5 \mathrm{~nm}$ in diameter have been synthesized by thermal decomposition of iron stearate in the presence of oleic acid in a high boiling solvent. ${ }^{28,29}$ A size selective precipitation (SSP) step is applied in order to reduce the size dispersion. The nanoparticle composition is close to that of maghemite as magnetite $\mathrm{Fe}_{3} \mathrm{O}_{4}$ oxidizes readily at such small sizes. Mössbauer spectroscopy under an applied field and magnetic measurements after cooling under an applied field demonstrated that these NPs display spin canting. In order to reduce the effect of dipolar interactions, nanoparticle dispersions in a polymer matrix $(\sim 0.1 \%$ wt./wt.) have been prepared by mixing the iron oxide NPs suspended in chloroform to a solution of PMMA in chloroform and by leaving the solvent to slowly evaporate in order to obtain a NP/polymer composite. $^{30}$

As for the Co nanoparticle sample, a similar procedure has been used to characterize magnetic $\mathrm{Fe}_{3-\mathrm{x}} \mathrm{O}_{4}$ nanoparticles. We emphasize that the magnetic anisotropy energy (MAE) expression implicitly supposes that the clusters size distribution is the major source of dispersion for the magnetization switching energy barrier. As a matter of fact, this assumption is not firmly justified, especially in the case of an oxide: in addition to the usual surface and shape distribution effects, spin canting can modify the MAE. Anyway, since the simple relation $\Delta E=K_{\text {eff }} . V$ appears to be satisfactory in the present case, we will not go further into a cluster anisotropy analysis in this study.

Figure 4 shows the good agreement between experiments and fit for the $\mathrm{ZFC} / \mathrm{FC}$ and $m(H)$ at $300 \mathrm{~K}$. In this case, The PDF distribution follows a Gaussian function

$$
P D F(D)=\frac{1}{\sigma D_{m} \sqrt{\pi / 2}} \exp \left[-2\left(\frac{D-D_{m}}{\sigma D_{m}}\right)^{2}\right],
$$

with a median magnetic diameter $D_{m \text { mag }}=5.4 \pm 0.3 \mathrm{~nm}$ in good agreement with the size distribution measured on TEM 

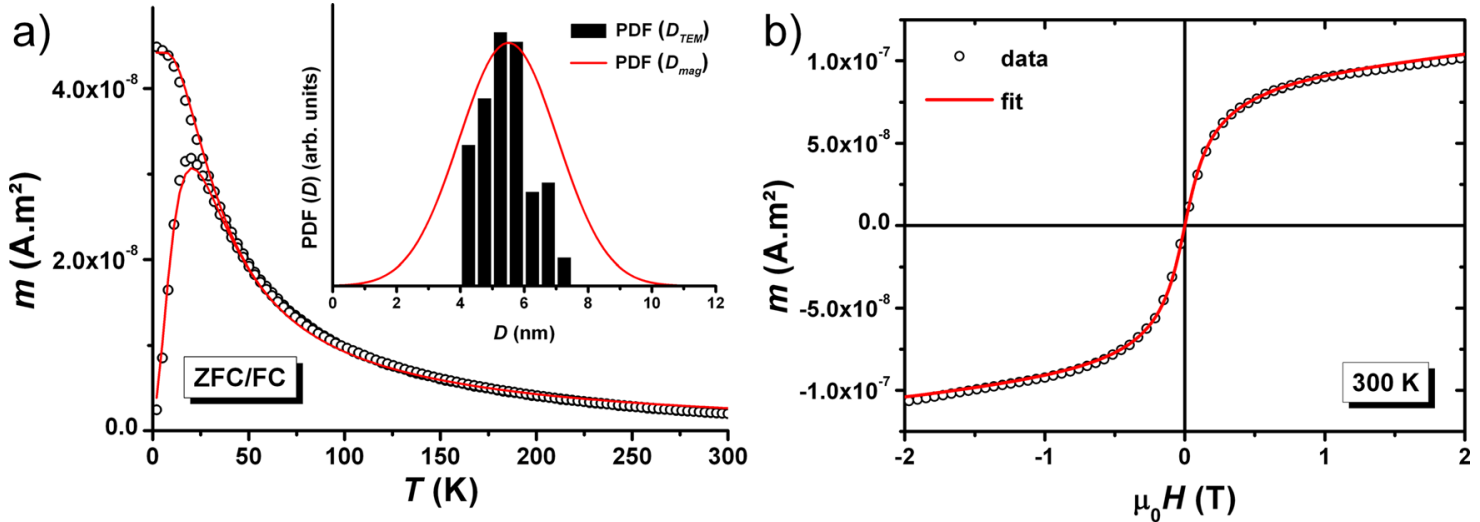

FIG. 4. ZFC/FC magnetization curves and $m(H)$ curve at $300 \mathrm{~K}$ for the iron oxide nanoparticle sample (a). The red lines correspond to the fit using the "triple fit." The size distributions as derived from the "triple fit" and from TEM observations are shown in (b).

micrographs and a standard deviation $\sigma_{\mathrm{mag}}=0.28 \pm 0.05$, larger than TEM (see Fig. 4 (inset)). This increase of $\sigma_{\text {mag }}$ may originate from the magnetization dispersion due to spin canting. ${ }^{31}$ The effective anisotropy is found to be $K_{\text {eff }}=31 \pm 4 \mathrm{~kJ} \mathrm{~m}^{-3}$ which is close to the bulk value.

Figure 5 shows the typical thermal variations of the real and imaginary part of the $\chi_{\mathrm{ac}}$. We observe the crossover from the blocked to the superparamagnetic regime with increasing temperature. Similarly, $T_{\max }$ increases as the frequency of measurement increases. All curves were fitted simultaneously using Eqs. (3) and (4) and the size distribution deduced from triple fit. We derive, in good agreement with the ZFC/FC triple fit, a value of $K_{\text {eff }}=25 \pm 4 \mathrm{~kJ} \mathrm{~m}^{-3}$ and a pre-exponential time $\tau_{0}=3.9 \times 10^{-10} \mathrm{~s}$. On the contrary, with the simple rule using the plot of the frequency versus $T_{\max }$ (Eq. (5)), we find $\tau_{0}=1.3 \times 10^{-10} \mathrm{~s}$ and $K_{\text {eff }}=41 \mathrm{~kJ} \mathrm{~m}^{-3}$, which overestimates the effective anisotropy constant.

\section{DISCUSSION AND CONCLUSION}

We have shown that $\tau_{0}$ and a precise $K_{\text {eff }}$ value can be obtained from $\chi_{\mathrm{ac}}$ experiments, if and only if the size distri-

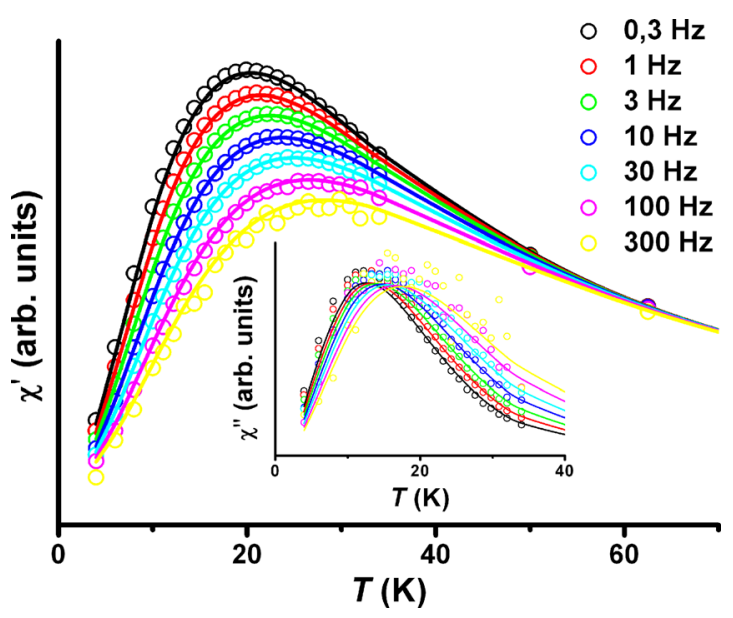

FIG. 5. Typical thermal variations of the real and imaginary part of acsusceptibility of the iron oxide nanoparticle sample, plotted for different frequencies. The solid line corresponds to the fit described in the text, using PDF deduced from the "triple fit." bution is determined from another technique such as TEM or triple fit. The satisfactory results in the description of experimental curves in that case allow concluding that this approach gives more reliable results than the simple model (plot of $-\ln \omega$ vs. $T_{\max }$ ) which neglects the size distribution and leads to a clear overestimation of $K_{\text {eff }}$. The fact that $K_{\text {eff }}$ deduced from the $\chi_{\mathrm{ac}}$ fits is very close to the triple fit estimated value is also an argument in favor of this approach. Moreover, combining ac and dc analysis reduces the uncertainties in the parameters simply by fitting a larger number of curves. Similar experimental procedures can be applied on different NPs based on physical or chemical way preparation. Finally, the reported experimental $\tau_{0}$ values are in the range usually reported in the literature around $10^{-9}$ to $10^{-12} \mathrm{~s}$. It is clear that $\tau_{0}$ depends on the intrinsic magnetic properties of the NP parameters (damping and temperature) and on their environment. To go further, it would be interesting to extend the range of the time measurement by using for instance ferromagnetic resonance or Mössbauer experiments.

${ }^{1}$ A. Moser, K. Takano, D. T. Margulies, M. Albrecht, Y. Sonobe, Y. Ikeda, S. Sun, and E. E. Fullerton, J. Phys. D: Appl. Phys. 35, R157-R167 (2002).

${ }^{2}$ S. Mornet, S. Vasseur, F. Grasset, and E. Duguet, J. Mater. Chem. 14, 2161 (2004).

${ }^{3}$ B. Basly, D. Felder-Flesch, P. Perriat, C. Billotey, J. Taleb, G. Pourroy, and S. Begin-Colin, Chem. Commun. (Cambridge) 46, 985-987 (2010).

${ }^{4}$ V. Skumryev, S. Stoyanov, Y. Zhang, G. Hadjipanayis, D. Givord, and J. Nogués, Nature 423, 850-853 (2003).

${ }^{5}$ L. Néel, C. R. Acad. Sci. Hebd Seances Acad. Sci. 228, 664-666 (1949).

${ }^{6}$ J. Dormann, F. D'Orazio, F. Lucari, E. Tronc, P. Prené, J. Jolivet, D. Fiorani, R. Cherkaoui, and M. Noguès, Phys. Rev. B 53, 14291-14297 (1996). ${ }^{7}$ C. Petit, J. Appl. Phys. 95, 4251 (2004).

${ }^{8}$ A. Tamion, M. Hillenkamp, A. Hillion, F. Tournus, J. Tuaillon-Combes, O. Boisron, S. Zafeiratos, and V. Dupuis, J. Appl. Phys. 110, 063904 (2011).

${ }^{9}$ A. Tamion, M. Hillenkamp, F. Tournus, E. Bonet, and V. Dupuis, Appl. Phys. Lett. 95, 062503 (2009).

${ }^{10}$ F. Tournus and E. Bonet, J. Magn. Magn. Mater. 323, 1109-1117 (2010).

${ }^{11} \mathrm{~F}$. Tournus and A. Tamion, J. Magn. Magn. Mater. 323, 1118-1127 (2010).

${ }^{12}$ F. Tournus, N. Blanc, A. Tamion, M. Hillenkamp, and V. Dupuis, Phys. Rev. B 81, 220405(R) (2010).

${ }^{13}$ A. Tamion, C. Raufast, M. Hillenkamp, E. Bonet, J. Jouanguy, B. Canut, E. Bernstein, O. Boisron, W. Wernsdorfer, and V. Dupuis, Phys. Rev. B 81, 144403 (2010).

${ }^{14}$ A. Hillion, A. Tamion, F. Tournus, J.-B. Flament, M. Hillenkamp, E. Bonet, and V. Dupuis, IEEE Trans. Magn. 47, 3154-3156 (2011). 
${ }^{15}$ M. Shliomis and V. I. Stepanov, J. Magn. Magn. Mater. 122, 176-181 (1993).

${ }^{16}$ J. L. García-Palacios, Advances in Chemical Physics (John Wiley \& Sons, Inc., Hoboken, NJ, USA, 1997).

${ }^{17}$ Y. L. Raikher and V. I. Stepanov, Phys. Rev. B 55, 15005-15017 (1997).

${ }^{18}$ J. Gittleman, B. Abeles, and S. Bozowski, Phys. Rev. B 9, 3891-3897 (1974).

${ }^{19}$ S. H. Masunaga, R. F. Jardim, and J. Rivas, Phys. Rev. B 80, 184428 (2009).

${ }^{20}$ J. Zhang, C. Boyd, and W. Luo, Phys. Rev. Lett. 77, 390-393 (1996).

${ }^{21}$ A. Perez, P. Melinon, V. Dupuis, L. Bardotti, B. Masenelli, F. Tournus, B. Prevel, J. Tuaillon-Combes, E. Bernstein, A. Tamion, N. Blanc, D. Tainoff, O. Boisron, G. Guiraud, M. Broyer, M. Pellarin, N. Del Fatti, F. Vallee, E. Cottancin, J. Lerme, J.-L. Vialle, C. Bonnet, P. Maioli, A. Crut, C. Clavier, J. L. Rousset, and F. Morfin, Int. J. Nanotechnol. 7, 523-574 (2010).

${ }^{22}$ V. L. Calero-Diaz Del Castillo and C. Rinaldi, IEEE Trans. Magn. 46, 852-859 (n.d.).
${ }^{23}$ C. Vázquez-Vázquez, M. A. López-Quintela, M. C. Buján-Núñez, and J. Rivas, J. Nanopart. Res. 13, 1663-1676 (2010).

${ }^{24}$ H. C. Bryant, N. L. Adolphi, D. L. Huber, D. L. Fegan, T. C. Monson, T. E. Tessier, and E. R. Flynn, J. Magn. Magn. Mater. 323, 767-774 (2011).

${ }^{25}$ D. Fiorani, A. M. Testa, F. Lucari, F. D’Orazio, and H. Romero, Physica B 320, 122-126 (2002).

${ }^{26}$ J. Lopez, H. Pfannes, R. Paniago, J. Sinnecker, and M. Novak, J. Magn. Magn. Mater. 320, e327-e330 (2008).

${ }^{27}$ R. Zheng, H. Gu, B. Zhang, H. Liu, X. Zhang, and S. P. Ringer, J. Magn. Magn. Mater. 321, L21-L27 (2009).

${ }^{28}$ M. Pauly, B. P. Pichon, P.-A. Albouy, S. Fleutot, C. Leuvrey, M. Trassin, J.-L. Gallani, and S. Begin-Colin, J. Mater. Chem. 21, 16018 (2011).

${ }^{29}$ A. Demortière, P. Panissod, B. P. Pichon, G. Pourroy, D. Guillon, B. Donnio, and S. Bégin-Colin, Nanoscale 3, 225-232 (2011).

${ }^{30}$ M. Pauly, B. P. Pichon, P. Panissod, S. Fleutot, P. Rodriguez, M. Drillon, and S. Begin-Colin, J. Mater. Chem. 22, 6343-6350 (2012).

${ }^{31}$ M. Di Marco, M. Port, P. Couvreur, C. Dubernet, P. Ballirano, and C. Sadun, J. Am. Chem. Soc. 128, 10054-10059 (2006). 